\title{
DETERMINACIÓN DE LOS MODELOS DE VELOCIDAD DE PARTÍCULAS EN VOLADURAS
}

\author{
(Particle Speed Determination Models in Blasting)
}

\section{Luis Humberto Pinto Morales*, María del Carmen Fuentes F.**}

\author{
*I.GeosoftMine, pintomoraleshumberto@yahoo.com \\ **Uptc-Sogamoso,marcaf_humb@latinmail.com
}

(Recibido Octubre 10 de 2006 y aceptado Abril 15 de 2007)

\section{Resumen:}

Se exponen los resultados obtenidos con la utilización de diferentes modelos de velocidad de las partículas empleados durante voladuras que se realizaron en la empresa Calizas y Agregados Boyacá S.A., para de esta forma estimar y controlar el nivel de daño generado por los trabajos que se ejecutan. Esta investigación se realizó con el objetivo de monitorear las vibraciones inducidas por las voladuras sobre las estructuras y edificaciones de interés cercanas a la línea de diseño de pit final de la cantera de caliza de esta empresa en Sáchica (Boyacá).

Palabras clave: Vibraciones, Voladuras, Acelerógrafos.
Abstract:

This is an exposition of the results obtained with the use of different particles speed models, used during the blasts carried out in the company "Calizas y Agregados Boyacá S.A.", in order to consider and to control the damage's level caused by the execution of these works. This investigation was made with the aim to monitor the vibrations induced by the blasts on the structures and constructions of interest, near the final design line of the limestone quarry pit, that belongs to this company, in Sáchica, Boyacá.

Key Words: Vibrations, Blasting, Acceleration Graphs Machines

\section{INTRODUCCIÓN}

$\mathbf{L}$ as vibraciones generadas por voladuras son producto directo del uso de explosivos para la fragmentación de la roca. La vibración del suelo o energía sísmica es comúnmente descrita como un desplazamiento con variación del tiempo, velocidad o aceleración de un punto particular (partícula) en el suelo, el cual puede generar molestias a personas o daños a estructuras cercanas a los sitios de explotación. La mina de caliza objeto de estudio se encuentra en el municipio de Sáchica, departamento de Boyacá, Colombia (ver figura 1).

\section{METODOLOGÍA}

El trabajo se desarrolló en cuatro etapas fundamentales:

- Toma de registros y cuantificación del comportamiento de la vibración con distancia y carga (ver figura 2).

- Ajuste estadístico o modelo de ecuación que describe dicho comportamiento.

- Ajuste de los modelos de vibración para permitir un uso confiable.

- Establecer criterios de daño.

A continuación se muestra un esquema del procedimiento por seguir durante la adquisición y registro de las ondas de choque generadas por las voladuras.

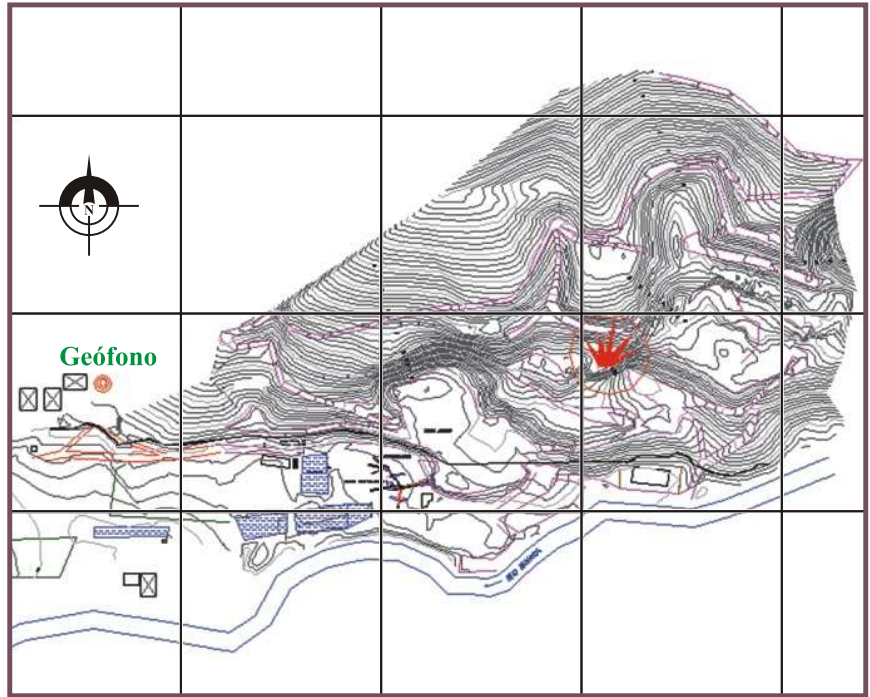

Figura 1. Localización del punto de explosión $\mathrm{y}$ el punto de registro.

\subsection{Medición de vibraciones (monitoreo y control)}

En este proceso se obtienen, para cada evento, los registros para los tres canales -longitudinal (L), vertical (V) y transversal (T)-, como los que se aprecian en la figura 3 . 


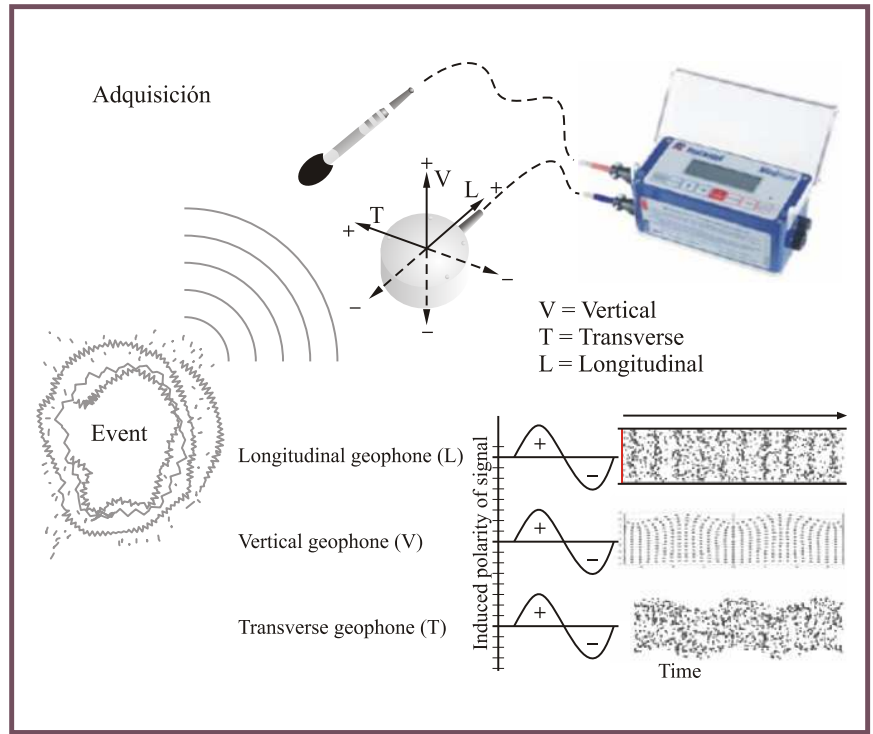

Figura 2. Equipo de registro Minimate plus DS 077, de fabricación Americana- Canadiense (USBM).

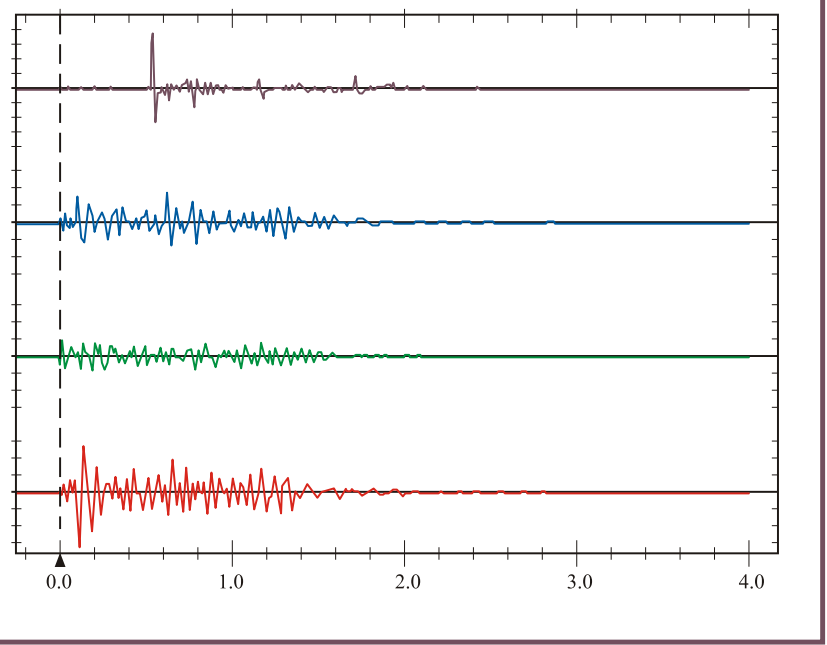

Figura 3. Registro de vibración y ondas de sobrepresión.

\subsection{Determinación de la ley de Vibración}

En la determinación de la ley de Vibración se deben tener en cuenta algunos conceptos importantes, que son explicados a continuación:

- Concepto de distancia escalada. Escalamiento: es la designación de la relación que correlaciona los niveles de movimiento del terreno, a distintas distancias de la voladura. Para ello se utiliza un factor de escalamiento, basado en parámetros dimensionales para la distancia. La distancia escalada se obtiene como una combinación de la distancia y del peso de la carga.
La energía total de la onda resultante de una columna de carga, donde la razón longituddiámetro es mayor a 6 , toma la forma de cilindro. El volumen de este cilindro de compresión varía directamente con el cuadrado de su radio. Así, el nivel peak del movimiento en un punto dado es inversamente proporcional al cuadrado de la distancia desde el tiro.

La fórmula de escalamiento, que relaciona el peak de la velocidad de partícula con la distancia escalada, ha sido desarrollada a través de los resultados obtenidos en terreno, usando equipos de monitoreo de vibraciones.

La distancia escalada $\left(\mathrm{D} / \mathrm{W}^{\wedge} 0,5\right)$ combina el efecto de la carga total por retardo (W) sobre el nivel inicial de choque, con el incremento de la distancia $\mathrm{D}$ desde la detonación al punto de medición.

Esta relación empírica involucra constantes del terreno, $\mathbf{k}$ y m, las cuales relacionan las características locales de la roca sobre la tasa de atenuación de la velocidad de partícula.

La atenuación geométrica está incluida en la pendiente exponencial, $\mathbf{m}$, de la siguiente ecuación:

$$
\mathrm{V}_{\text {máx }}=\mathrm{k} *\left(\mathrm{D} / \mathrm{W}^{0.5}\right)^{\mathrm{m}}
$$

donde:

$\mathrm{V}=$ Peak de velocidad de partícula $(\mathrm{mm} / \mathrm{s})$

$\mathrm{D}=$ distancia entre tiro y lugar de registro $(\mathrm{m})$

$\mathrm{W}=$ peso total de explosivo por retardo $(\mathrm{Kg})$

$\mathrm{K}, \mathrm{m}=$ constantes del terreno

$\left(\mathrm{D} / \mathrm{W}^{0.5}\right)^{\mathrm{m}}=$ distancia escalada para cargas cilíndricas

Las constantes del terreno son determinadas de un gráfico logarítmico de la velocidad de partícula vs. la distancia escalada, donde $\mathrm{m}$ corresponde a la pendiente $\mathrm{y} \mathrm{k}$ es el intercepto a la distancia escalada de 1 .

- Modelos de velocidad de partícula. La literatura presenta diversos criterios de velocidad, entre los cuales destacan:

$$
\begin{array}{ll}
\text { Devine } & \mathrm{V}=\mathrm{k} *\left(\mathrm{D} / \mathrm{W}^{0.5}\right)^{\mathrm{m}} \\
\text { Hendrom } & \mathrm{V}=\mathrm{k} *\left(\mathrm{D} / \mathrm{W}^{0.33}\right)^{\mathrm{m}} \\
\text { Langerfors } & \mathrm{V}=\mathrm{k} *\left(\mathrm{D} / \mathrm{W}^{1.5}\right)^{\mathrm{m}}
\end{array}
$$

\section{- Criterio coeficiente escalar}

Criterio General Exponencial

$$
D=\left(\frac{d}{W 1 / 2}\right) * e\left(\frac{d^{*} \beta}{\alpha}\right)
$$

Criterio General

$$
D=\left(\frac{d}{W \beta / \alpha}\right)
$$


Langefors (1963)

$$
D=\left(\frac{d}{W^{3} / 2}\right)
$$

Hendron (Bulletin 656)

$$
D=\left(\frac{d}{W^{1 / 3}}\right)
$$

Devine (1966)

$$
D=\left(\frac{d}{W^{1 / 2} / 2}\right)
$$

El más utilizado en minería a tajo abierto es el Modelo de Devine; el cual se tuvo en cuenta para el presente estudio:

$$
P P V=K^{*}\left(\frac{d}{W 1 / 2}\right)^{\alpha}
$$

Donde:

PPV: velocidad de partícula Peak $(\mathrm{mm} / \mathrm{s})$

$\mathrm{W}$ : peso de la carga explosiva

$\mathrm{d}$ : distancia entre el punto de medición y la carga explosiva detonada

$\mathrm{K}$ : factor de velocidad

a: factor de atenuación

Teóricamente, este criterio es el que mejor representa el comportamiento de la vibración en el campo lejano para cargas cilíndricas, donde el análisis dimensional sugiere que las distancias deben ser corregidas dividiéndolas por la raíz cuadrada de la carga.

De ellos, el más utilizado, por investigadores, usuarios y empresas, es el Criterio de Devine.

A continuación se presentan los resultados de los ajustes obtenidos al modelar una ley de Vibración con la información obtenida de las 7 voladuras, realizadas en la cantera de estudio.

\subsection{Criterio de prevención de daños}

La finalidad es establecer límites de vibración y cargas máximas operantes vs. distancia, para la protección a estructuras cercanas a la mina y controlar posible sobrequiebre del macizo.

- Estructuras: para las estructuras, la velocidad crítica se limita a $31,8 \mathrm{~mm} / \mathrm{s}$, entre 0 y 91 metros de distancia del punto de explosión y el punto de muestreo -según norma Americana OSM (Oficina de Minería de Superficie)-.
- Ley de Vibración: aplicando el criterio de Devine, se obtiene el siguiente modelo de ajuste para el Sector Norte.

Coeficiente de correlación: 0,841

Coeficiente k: 959310

Coeficiente alfa: -1056

Puntos de ajuste: 7,00

La ley de Vibración se calculará así:

$$
\mathrm{V}=959310 *\left(\mathrm{D} / \mathrm{W}^{0.5}\right)^{-1.056}
$$

Con fines de diseño, debe adoptarse un modelo que asegure la mayor concentración posible de puntos cercanos al ajuste.

Para ello, debe desplazarse la recta hacia arriba, incrementando el valor de k. Así, el valor de k es de 1453 297, lo que asegura el $80 \%$ de los puntos medidos.

El modelo ajustado será:

$$
\mathrm{V}=1453297 *\left(\mathrm{D} / \mathrm{W}^{0.5}\right)^{-1.056}
$$

Aplicando como criterio de daño, un límite de velocidad de partícula de $31,8 \mathrm{~mm} / \mathrm{s}$ para la estructura a cautelar, podemos diseñar la carga requerida de explosivo para el límite de vibraciones establecido.

Determinaron inicialmente, la distancia, carga explosiva y velocidad peak para 7 voladuras monitoreadas, y se ajustó la regresión correspondiente (ver tablas 1 y 2 )

Tabla 1. Datos de las voladuras monitoreadas.

\begin{tabular}{|c|c|c|c|c|c|c|}
\hline Dist & Explosivo & $\begin{array}{c}\text { (Raíz cuadrada) } \\
\text { de }\end{array}$ & $\begin{array}{c}\text { Transversal } \\
\text { Peak }\end{array}$ & $\begin{array}{c}\text { Vertical } \\
\text { Peak }\end{array}$ & $\begin{array}{c}\text { Longitudinal } \\
\text { Peak }\end{array}$ & $\begin{array}{c}\text { PPV } \\
\text { Peak }\end{array}$ \\
\hline $\mathbf{( m )}$ & & $(\mathbf{m} / \mathbf{k g} \mathbf{1} / \mathbf{2}$ & $\mathbf{( m m} / \mathbf{s})$ & $\mathbf{( m m} / \mathbf{s})$ & $\mathbf{( m m} / \mathbf{s})$ & $(\mathbf{m m} / \mathbf{s})$ \\
\hline & & & & & & \\
\hline 20 & 1.7 & 15.34 & 30.80 & 30.10 & 29.00 & 30.80 \\
\hline 50.0 & 1.7 & 38.35 & 29.00 & 13.50 & 29.70 & 29.70 \\
\hline 100 & 1.7 & 76.70 & 17.90 & 14.90 & 16.10 & 17.90 \\
\hline 150 & 1.7 & 115.04 & 9.56 & 2.86 & 5.38 & 9.56 \\
\hline 200 & 1.7 & 153.39 & 3.75 & 2.59 & 3.65 & 3.75 \\
\hline 250.0 & 1.7 & 191.74 & 1.78 & 1.52 & 3.05 & 3.05 \\
\hline 300.0 & 1.5 & 244.95 & 1.94 & 1.52 & 1.95 & 1.95 \\
\hline
\end{tabular}

Tabla 2. Datos de ajuste de regresión al 80\%.

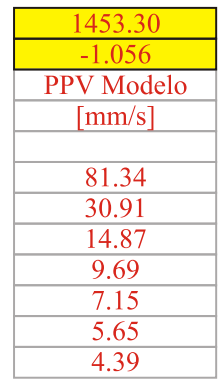

\begin{tabular}{|c|c|}
\hline Pendiente & -1.056 \\
\hline K Promedio & 959.310 \\
\hline K - 80\% & 1453.297 \\
\hline Correlación (R2) & 0.841 \\
\hline Puntos & 7 \\
\hline
\end{tabular}

Los datos obtenidos permitieron ajustar el modelo de vibraciones aplicando el modelo Devine (ver figura 4). 


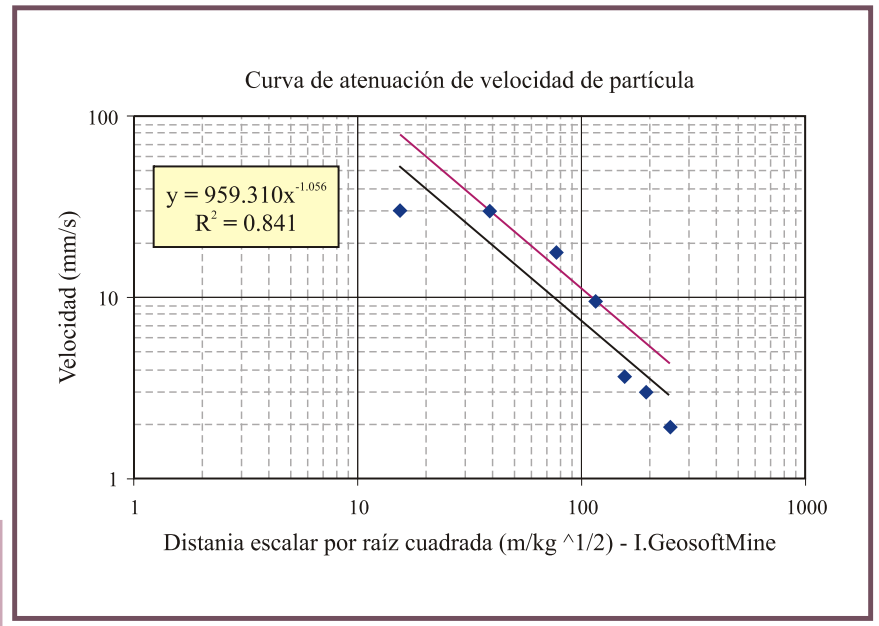

Figura 4. Curvas de atenuación.

\section{RESULTADOS}

El procesamiento de los datos obtenidos durante los monitoreos de las voladuras permitió obtener los siguientes resultados:

- Ábaco de diseño sector norte para vibraciones esperadas con diferentes cargas explosivas: el ábaco permite calcular la vibración esperada a diferentes distancias y para $0.1,0.5,1.0$, $1.5,2.0$ y $2.5 \mathrm{~kg}$ de explosivo (ver figura 5 y tabla 3 ).

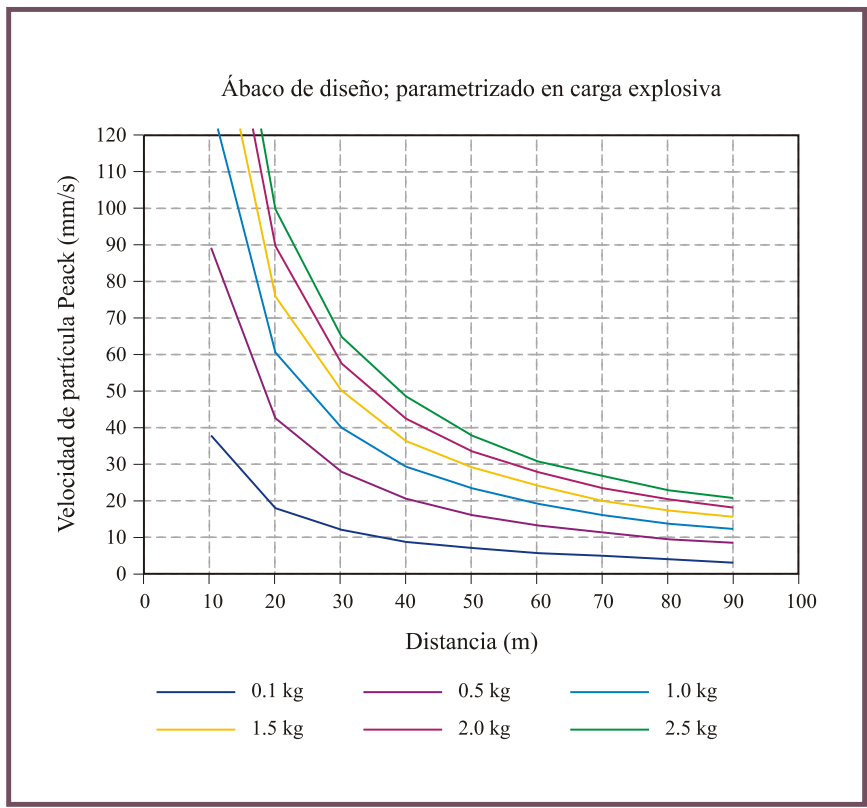

Figura 5. Ábaco de diseño sector norte con diferentes cargas explosivas.
Tabla 3. Datos de las voladuras monitoreadas.

\begin{tabular}{|c|c|c|c|c|c|c|}
\hline $\mathbf{K}$ & 1453.30 & \multirow{2}{*}{\multicolumn{2}{|c|}{$\begin{array}{c}\text { Modelo } \\
\text { DEVINE } \\
\text { V (1/2) }\end{array}$}} & & & \\
\hline Alfa & -1.055843 & & & & & \\
\hline Kilos & $0-1$ & 0.5 & 1.0 & 1.5 & 2.0 & 2.5 \\
\hline Dist & & & & & & \\
\hline (m) & & & & & & \\
\hline 10 & $\begin{array}{l}37.9 \\
\end{array}$ & 88.6 & 127.8 & 158.3 & 184.3 & 207.3 \\
\hline 20 & 18.2 & 42.6 & 61.5 & 76.1 & 88.6 & 99.7 \\
\hline 30 & 11.9 & 27.8 & 40.1 & 49.6 & 57.8 & 65.0 \\
\hline 40 & 8.8 & 20.5 & 29.6 & 36.6 & 42.6 & 48.0 \\
\hline 50 & 6.9 & 16.2 & 23.4 & 28.9 & 33.7 & 37.9 \\
\hline 60 & 5.7 & 13.4 & 19.3 & 23.9 & 27.8 & 31.3 \\
\hline 70 & 4.9 & 11.4 & 16.4 & 20.3 & 23.6 & 26.6 \\
\hline 80 & 4.2 & 9.9 & 14.2 & 17.6 & 20.5 & 23.1 \\
\hline 90 & 3.7 & 8.7 & 12.6 & 15.6 & 18.1 & 20.4 \\
\hline
\end{tabular}

La tabla 3 permite calcular la velocidad crítica para diferentes cargas explosivas y a una distancia dada. Por ejemplo, para un carga de $2 \mathrm{~kg}$ y a una distancia de 60 metros se obtendría una velocidad de partícula de $27,8 \mathrm{~mm} / \mathrm{s}$.

Se diseñó además el ábaco del sector norte, para carga explosiva en un nivel de vibración determinado (ver figura 6).

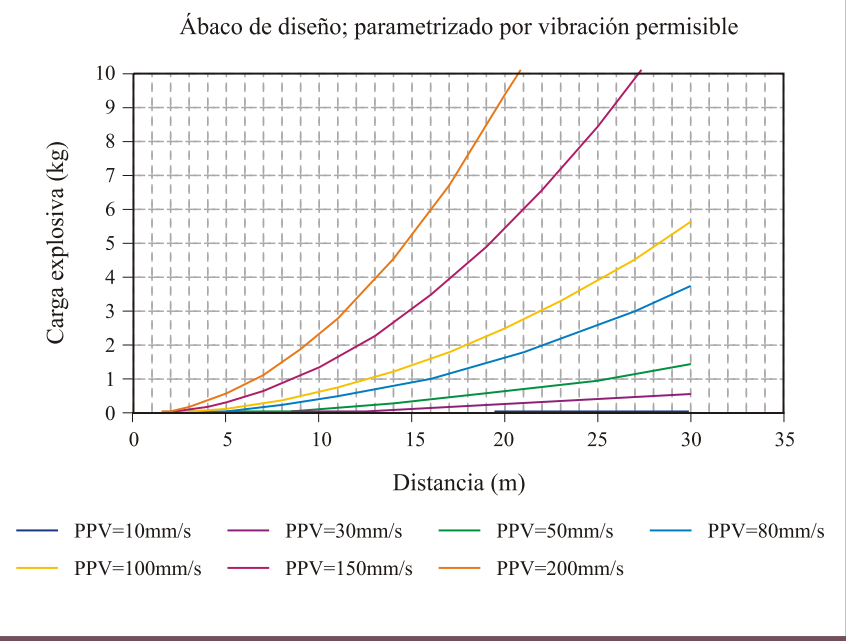

Figura 6. Ábaco de diseño sector norte.

Las voladuras monitoreadas con cargas explosivas, recomendadas para diferentes límites de vibración fueron: 10, 30, 50, $80,100,150$ y $200 \mathrm{~mm} / \mathrm{seg}$ (ver tabla 4), permitió calcular la cantidad de explosivo por unidad de retardo que se puede utilizar para no superar el umbral seguro de la velocidad de partícula. Por ejemplo: para que una voladura no supere los 30 $\mathrm{mm} / \mathrm{s}$, a un a distancia de 20 metros, se recomienda utilizar una carga explosiva de $0,26 \mathrm{~kg}$. 
Tabla 4. Datos de las voladuras monitoreadas.

\begin{tabular}{|c|c|c|c|c|c|c|c|}
\hline $\mathbf{K}$ & 1453.30 & & & \multirow{2}{*}{$\begin{array}{c}\text { Modelo } \\
\text { DEVINE } \\
\text { V (1/2) }\end{array}$} & & & \\
\hline Alfa & -1.055843 & & & & & & \\
\hline PPV & 10.0 & 30.0 & 50.0 & 80.0 & 100.0 & 150.0 & 200.0 \\
\hline Dist & \multicolumn{7}{|c|}{ Carga Explosiva (W) } \\
\hline (m) & \multicolumn{7}{|c|}{$(\mathrm{kg})$} \\
\hline 1 & 0.00 & 0.00 & 0.00 & 0.00 & 0.01 & 0.01 & 0.02 \\
\hline 2 & 0.00 & 0.00 & 0.01 & 0.02 & 0.03 & 0.05 & 0.09 \\
\hline 3 & 0.00 & 0.01 & 0.02 & 0.04 & 0.06 & 0.12 & 0.21 \\
\hline 4 & 0.00 & 0.01 & 0.03 & 0.07 & 0.10 & 0.22 & 0.37 \\
\hline 5 & 0.00 & 0.02 & 0.04 & 0.10 & 0.16 & 0.34 & 0.58 \\
\hline 6 & 0.00 & 0.02 & 0.06 & 0.15 & 0.23 & 0.49 & 0.84 \\
\hline 7 & 0.00 & 0.03 & 0.08 & 0.20 & 0.31 & 0.66 & 1.14 \\
\hline 8 & 0.01 & 0.04 & 0.11 & 0.26 & 0.40 & 0.87 & 1.49 \\
\hline 9 & 0.01 & 0.05 & 0.14 & 0.33 & 0.51 & 1.10 & 1.89 \\
\hline 10 & 0.01 & 0.06 & 0.17 & 0.41 & 0.63 & 1.35 & 2.34 \\
\hline 11 & 0.01 & 0.08 & 0.20 & 0.50 & 0.76 & 1.64 & 2.83 \\
\hline 12 & 0.01 & 0.09 & 0.24 & 0.59 & 0.90 & 1.95 & 3.36 \\
\hline 13 & 0.01 & 0.11 & 0.29 & 0.70 & 1.06 & 2.29 & 3.95 \\
\hline 14 & 0.02 & 0.13 & 0.33 & 0.81 & 1.23 & 2.65 & 4.58 \\
\hline 15 & 0.02 & 0.14 & 0.38 & 0.93 & 1.41 & 3.05 & 5.26 \\
\hline 16 & 0.02 & 0.16 & 0.43 & 1.05 & 1.61 & 3.47 & 5.98 \\
\hline 17 & 0.02 & 0.19 & 0.49 & 1.19 & 1.82 & 3.91 & 6.75 \\
\hline 18 & 0.03 & 0.21 & 0.55 & 1.33 & 2.04 & 4.39 & 7.57 \\
\hline 19 & 0.03 & 0.23 & 0.61 & 1.49 & 2.27 & 4.89 & 8.43 \\
\hline 20 & 0.03 & 0.26 & 0.68 & 1.65 & 2.51 & 5.42 & 9.34 \\
\hline 21 & 0.04 & 0.28 & 0.75 & 1.82 & 2.77 & 5.97 & 10.30 \\
\hline 22 & 0.04 & 0.31 & 0.82 & 1.99 & 3.04 & 6.56 & 11.31 \\
\hline 23 & 0.04 & 0.34 & 0.89 & 2.18 & 3.32 & 7.17 & 12.36 \\
\hline 24 & 0.05 & 0.37 & 0.97 & 2.37 & 3.62 & 7.80 & 13.45 \\
\hline 25 & 0.05 & 0.40 & 1.06 & 2.57 & 3.93 & 8.47 & 14.60 \\
\hline 26 & 0.05 & 0.43 & 1.14 & 2.78 & 4.25 & 9.16 & 15.79 \\
\hline 27 & 0.06 & 0.47 & 1.23 & 3.00 & 4.58 & 9.87 & 17.03 \\
\hline 28 & 0.06 & 0.50 & 1.33 & 3.23 & 4.93 & 10.62 & 18.31 \\
\hline 29 & 0.07 & 0.54 & 1.42 & 3.46 & 5.28 & 11.39 & 19.65 \\
\hline 30 & 0.07 & 0.58 & 1.52 & 3.71 & 5.66 & 12.19 & 21.02 \\
\hline
\end{tabular}

- Cálculo de la velocidad crítica, para evitar algún fracturamiento del macizo: para este proceso se tuvo en cuenta la velocidad crítica en $\mathrm{mm} / \mathrm{s}$, donde se pueden generar daños o sobrefracturamiento del macizo rocoso según los criterios de daño de Holmberg y Persson (ver tabla 5).

Tabla 5. Tipos de daño según el PPV crítico.

\begin{tabular}{|l|c|}
\hline Criterios de daño & PPVcrítico \\
\hline Dilatación de fracturas & $1 / 4$ \\
\hline Aparición de nuevas grietas o fracturas & PPVcrítico \\
\hline Daño notorio u obvio & 4 \\
\hline Sobrequiebre & 8 \\
\hline
\end{tabular}

El criterio de Holmberg y Persson tiene en cuenta las características de las rocas como se muestra en la tabla 6.
Tabla 6. Parámetros físico-mecánicos de la roca caliza.

\begin{tabular}{|l|c|}
\hline Resistencia a la compresión (MPa) & 63 \\
\hline Resistencia a la tracción(MPa) & 6,3 \\
\hline Densidad $\left(\mathrm{t} / \mathrm{m}^{3}\right)$ & 2,7 \\
\hline Módulo de Young dinámico $(\mathrm{GPa})$ & 51,1 \\
\hline Velocidad de onda P(m/s) & 3700 \\
\hline
\end{tabular}

Aplicando la fórmula 12 se calculó el PPV crítico para medir el nivel correspondiente de daño (ver tabla 7).

$$
P P V_{\max }=\frac{\sigma_{T} \cdot V_{p}}{E}
$$

Tabla 7. Resultados del análisis.

\begin{tabular}{|l|c|c|}
\hline Nivel de daño & PPV crítico & $\mathbf{~ m m / s}$ \\
\hline Dilatación de fracturas & $1 / 4$ & 114 \\
\hline Aparición de nuevas grietas o fracturas & $\mathrm{PPV}$ & 456 \\
\hline Daño notorio u obvio & 4 & 1824 \\
\hline Sobrequiebre & 8 & 3648 \\
\hline
\end{tabular}

\section{CONCLUSIONES}

Para la estructura a cautelar, la velocidad crítica se limita a $31,8 \mathrm{~mm} / \mathrm{s}$, entre 0 y 91 metros de distancia del punto de explosión y el punto de muestreo, según norma Americana para la protección de estructuras OSM, Oficina de Minería de Superficie.

La velocidad crítica para evitar sobrefracturamiento del macizo rocoso es $P P V_{\text {CRIT }}=114 \mathrm{~mm} / \mathrm{s}$.

Los ábacos de diseño obtenidos según la ley de Vibración local, permitió realizar diseños técnicos de voladuras controladas, los cuales aseguran que el umbral establecido de $31,8 \mathrm{~mm} / \mathrm{seg}$ no se ha superado.

\section{BIBLIOGRAFÍA}

Allard, P. (1986). Etude des vibrations Engendrées par les Tirs de Mines", Scetaroute. Février.

ASP. Blastronics, Dr. Carlos Scherpenisse, Adamson W. R. (1997). Asesoría técnica en monitoreo de vibraciones para el modelamiento y control de daño. 
Barkley et ál. (1983). Ground and Air Vibrations Caused by Surface Blasting. N.T.I.S.

Bollinger, G. A. (1971). Blast Vibration Analysis.

Carvajal, A. (2006). Control de vibraciones producidas por voladuras. Chile: Universidad La Serena.

Esteves, J. M. (1978). Control of Vibrations Caused by Blasting, Memoria 498, Laboratorio Nacional de Engenharía Civíl, Lisboa.
Gama, Dinis C. (2004). Vibraçoes dos terrenos: da generaçao ao controle, I Semana Iberoamericana de Minas, Portugal. Pp. 147-152.

Hinzen, K. G. et al. (1987). A New Approach to Predict and Reduced Blast Vibration by Modelling of Acelerograms and Using a New Electronic Initiation System. S.E.E.

U.S. Bureau of Mines-RI 8506 Blast vibration Instrumentation. U.S. Bureau of Mines-RI 9455 Blast vibration impacting a distant community. 